\title{
DISTRIBUTION OF PLANETARY NEBULAE PERPENDICULAR TO THE DISK
}

\author{
D.C.V. MALLIK and S. CHATTERJEE
}

Indian Institute of Astrophysics, Bangalore 560034, India

From an analytical solution to the Boltzmann-Poisson equations for a thin, vertically isothermal self-gravitating disk with the assumptions that the mass spectrum of stars is expressible as an inverse power-law and the velocity dispersion perpendicular to the plane follows a law of the form $\left\langle V_{z}^{2}(m)>=V_{o}^{2}\right.$ for $m<m *$ and $\left\langle V_{z}^{2}(m)>=V_{o}^{2}(m * / m)^{\theta}\right.$ for $m \geq m *$, we have obtained a vertical height distribution of planetary nebulae : $n_{P N}(z)=\int_{m}^{m_{u}} n(m, z) \tau_{P N} / \tau(m) d m$, where $m_{u}=7.0 m_{\odot}, m_{l}=1.0 m_{\odot}$ and $n(m, z)=n(m, o) \exp \left(-\phi(z) /<V_{z}^{2}(m)>\right), \phi(z)$ being the potential at $z$. Figure 1 shows a normalised height distribution for various values of $V_{o}$ where we have assumed a Salpeter slope, a $\rho(o)=0.10 M_{\odot} p c^{-3}$ and set $m *=m_{l}=1.0 m_{\odot}$. Figure 2 shows filled circles obtained from the observational sample of Daub (1982, ApJ 260, 612) superposed on the theoretical distributions. Although there is agreement for large values of $z$, closer to the plane the observational sample falls below the theoretical curves. Since the observational sample is size-limited, we may be missing a larger fraction of small nebulae closer to the plane. It is also possible a single value of $V_{o}$ is not a correct representation of reality as these nebulae originate from stars of greatly differing ages and $V_{o}$ may have changed over the lifetime of the Galactic disk.
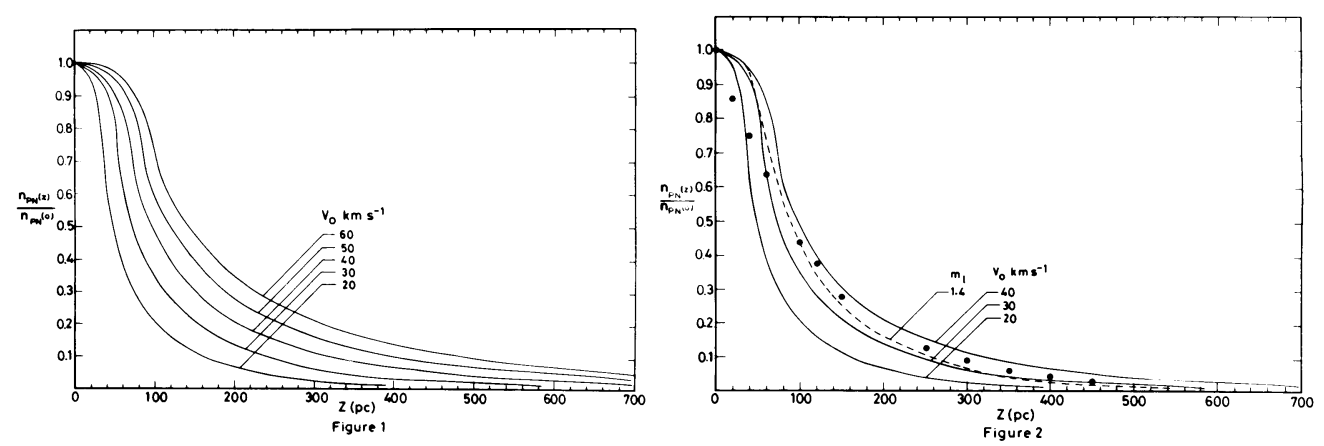